\title{
The charge transport mechanism and electron trap nature in thermal oxide on silicon
}

\author{
Damir R. Islamov, 1, 2, a) Vladimir A. Gritsenko,, ${ }^{1,2}$ Timofey V. Perevalov, ${ }^{1,2}$ Oleg M. Orlov, ${ }^{3}$, b) and Gennady Ya. \\ Krasnikov ${ }^{3}$ \\ 1) Rzhanov Institute of Semiconductor Physics, Siberian Branch of the Russian Academy of Sciences, \\ Novosibirsk 630090, Russian Federation \\ ${ }^{2)}$ Novosibirsk State University, Novosibirsk 630090, Russian Federation \\ 3) JSC Molecular Electronics Research Institute, Zelenograd, Moscow 124460, \\ Russian Federation
}

(Dated: 27 March 2018)

\begin{abstract}
The charge transport mechanism of electron via traps in amorphous $\mathrm{SiO}_{2}$ has been studied. Electron transport is limited by phonon-assisted tunneling between traps. Thermal and optical trap energies $W_{\mathrm{t}}=1.6 \mathrm{eV}$, $W_{\text {opt }}=3.2 \mathrm{eV}$, respectively, were determined. Charge flowing leads to oxygen vacancies generation, and the leakage current increases due to the increase of charge trap density. Long-time annealing at high temperatures decreased the leakage current to initial values due to oxygen vacancies recombination with interstitial oxygen. It is found that the oxygen vacancies act as electron traps in $\mathrm{SiO}_{2}$.
\end{abstract}

PACS numbers: 72.20.Jv, 77.55.df, 73.50.-h, 72.10.Di

Keywords: silica, oxygen vacancy, traps, charge transport, leakage current.

Silica $\mathrm{SiO}_{2}$ is the key material in electronic and optical devices, fibers. Intrinsic defects in $\mathrm{SiO}_{2}$ act as localization centers for electrons and holes (traps). The presence of such defects in particular layers of a device results in the whole device degradation. The main intrinsic defects in $\mathrm{SiO}_{2}$ are threefold coordinated silicon atom with an unpaired electron $(\mathrm{Si} .)^{1-3}$, the oxygen vacancy $(\mathrm{Si}-\mathrm{Si}$ bond $)^{4-9}$, non-bridging oxygen $(\mathrm{Si}-\mathrm{O} .)^{4,10}$, peroxide radical (Si-O-O. $)^{4,6}$ and a peroxide bridge (Si-O-O-Si) ${ }^{4}$. Here the sign $(-)$ shows a chemical bond formed by two electrons, the sign (.) denotes an unpaired electron. On the base of numerous experiments it was found that oxygen vacancies in $\mathrm{SiO}_{2}$ act as traps for holes ${ }^{6,9,11}$. According to the results of quantum-chemical simulations, it was predicted that the oxygen vacancy can act as traps for electrons ${ }^{10,12}$. However, the electron trapping energy and the trap ionization mechanism in strong electric field are unknown.

Long-time charge flowing through $\mathrm{SiO}_{2}$ in a strong electric field $\left(F \approx 10^{7} \mathrm{~V} / \mathrm{cm}\right.$ ) leads to the oxide conductivity increase at lower electric field values $(\approx 2 \times$ $\left.10^{6} \mathrm{~V} / \mathrm{cm}\right)$. Thus, an extra current through the dielectric is added to the Fowler-Nordheim tunneling current ${ }^{13,14}$, and addition values are comparable to the tunneling ones. This phenomenon is called Stress Induced Leakage Current $(\mathrm{SILC})^{4-7}$. Despite the fact that SILC is studied widely, both experimentally and theoretically ${ }^{15-18}$, the nature of the defects responsible for SILC and charge transport mechanism are still debatable questions. The purposes of the present study are identification of the ionization mechanism of electron traps in $\mathrm{SiO}_{2}$ in external electric field and determination of the trap parameters

\footnotetext{
a) Electronic mail: damir@isp.nsc.ru

b) Electronic mail: oorlov@mikron.ru
}

(ionization energy, concentration). The phenomenon of SILC was used to generate the traps in $\mathrm{SiO}_{2}$ followed by a charge transport study.

SILC and transport measurements were performed on FET transistors with the floating gate, manufactured using the $180 \mathrm{~nm}$ design rule technology. The $p$-Si substrate was used as the bottom contact, the $n^{+}$-type polySi floating gate was used as the top contact. The tunnel $\mathrm{SiO}_{2}$ layer thickness was $7.5 \mathrm{~nm}$. Test samples were formed of 16 parallel-connected $n$-Si/ $\mathrm{SiO}_{2} /$ poly-Si capacitors with the total area of the poly-silicon electrodes of $8 \times 10^{4} \mu \mathrm{m}^{2}$. SILC was generated by the current of $1 \mathrm{~mA} / \mathrm{cm}^{2}$. The total value of the flown charge via tunnel $\mathrm{SiO}_{2}$ was $0.01-10 \mathrm{C} / \mathrm{cm}^{2}$. Transport measurements have been performed at temperatures of $25-70{ }^{\circ} \mathrm{C}$.

Simulation was conducted for a 72-atom $\alpha-\mathrm{SiO}_{2}$ supercell with the Quantum-ESPRESSO software within the scope of the density functional theory with B3LYP hybrid functional ${ }^{19}$. This method yields the $\alpha-\mathrm{SiO}_{2}$ band gap of $8.0 \mathrm{eV}$, in excellent agreement with the experimental value $\mathrm{e}^{20}$ and with the theoretical value of $8.1 \mathrm{eV}$ obtained for amorphous $\mathrm{SiO}_{2}$ within framework of similar approach $^{3}$. See supplemental material $\mathrm{at}^{21}$ for detailed description of the numeric simulation procedure. [give brief description of material]. Electron and hole trap energies $W_{\mathrm{t}}^{\mathrm{e}}$ and $W_{\mathrm{t}}^{\mathrm{h}}$ at the $\mathrm{Si}-\mathrm{Si}$ bond are evaluated as difference between perfect and defect supercell electron affinities and ionization energies as follows:

$$
W_{\mathrm{t}}^{\mathrm{e} / \mathrm{h}}=E_{\mathrm{p}}^{+1 /-1}-E_{\mathrm{d}}^{+1 /-1}-E_{\mathrm{p}}^{0}+E_{\mathrm{d}}^{0}
$$

Here $E_{\mathrm{p}}^{0 /+1 /-1}$ and $E_{\mathrm{d}}^{0 /+1 /-1}$ represent the values for the total energy of an ideal and defect supercell with a different amount of the total charge. This approach was proposed for $\mathrm{SiO}_{2}$ to obtain the defect level positions between different charge states ${ }^{22}$, and used for charge localization energy calculation in $\mathrm{Si}_{3} \mathrm{~N}_{4}{ }^{23}$ and $\mathrm{HfO}_{2}{ }^{24}$. 


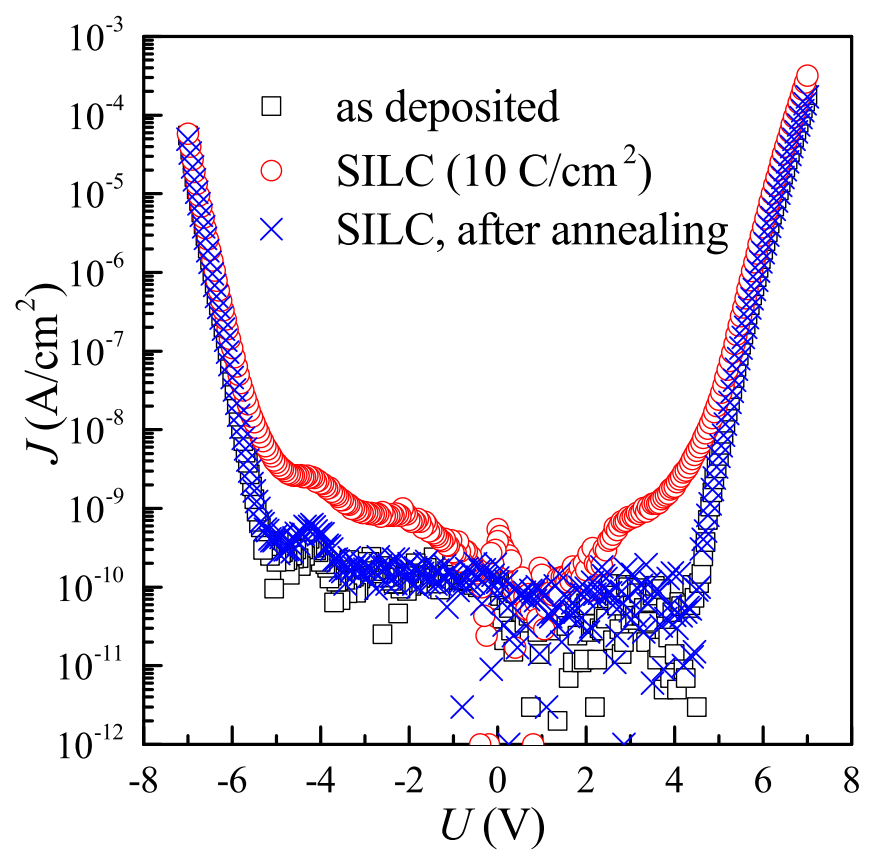

FIG. 1: (Color online) Experimental current-voltage characteristics of $n$ - $\mathrm{Si} / \mathrm{SiO}_{2} /$ poly-Si structures at room temperature $\left(25^{\circ} \mathrm{C}\right)$ before SILC $(\square)$, after SILC (o), and after SILC followed by long-time annealing $(x)$.

Experimental current-voltage characteristics $(J-V)$ of the as deposited oxide are shown in Fig. 1 by box symbols. At high voltages $|U|>5 \mathrm{~V}(F>6 \mathrm{MV} / \mathrm{cm})$ the current depends on the voltage exponentially and is limited by the tunnelling emission that proceeds by the Fowler-Nordheim mechanism ${ }^{13}$ :

$$
\begin{gathered}
J_{\mathrm{FN}}=A F^{2} \exp \left(-\frac{B \sqrt{m^{*}} \Phi_{0}^{3 / 2}}{F}\right), \\
A=\frac{e^{3}}{8 \pi h \Phi_{0}}, B=\frac{8 \pi \sqrt{2}}{3 h e},
\end{gathered}
$$

where $J_{\mathrm{FN}}$ is the tunneling current density, $m^{*}$ is the electron effective tunneling mass, $\Phi_{0}$ is the height of the triangular potential barrier for electrons at the $\mathrm{Si} / \mathrm{SiO}_{2}$ interface, $e$ is the elementary (electron) charge, $h$ is the Planck constant. When $|U|<5 \mathrm{~V}$, the measured current values will be determined by the sensitivity of the measuring devices and bulk properties of the substrate. According to photo emission measurements, the height of the triangular potential barrier for electrons at the $\mathrm{Si} / \mathrm{SiO}_{2}$ interface is $\Phi=3.14 \mathrm{eV}^{25}$. The electron energy spectrum quantization in high fields at the $\mathrm{Si} / \mathrm{SiO}_{2}$ interface leads to a descrease of the effective barrier height to $\Phi_{0}=2.9 \mathrm{eV}^{26}$. Taking $\Phi_{0}=2.9 \mathrm{eV}$ for simulations of experimental data with Eq. (2), we get the electron effective mass in $\mathrm{SiO}_{2} m^{*} / m_{\mathrm{e}}=0.5 \pm 0.02$ at both positive and negative biases on the poly-Si electrode. These results are in consistent with the literature ${ }^{26,27}$

The experimental $J-V$ characteristics after SILC $\left(10 \mathrm{C} / \mathrm{cm}^{2}\right)$ are shown in Fig. 1 by round symbols. At high bias $|U|>5 \mathrm{~V}$ on the poly-Si contact, the cur-

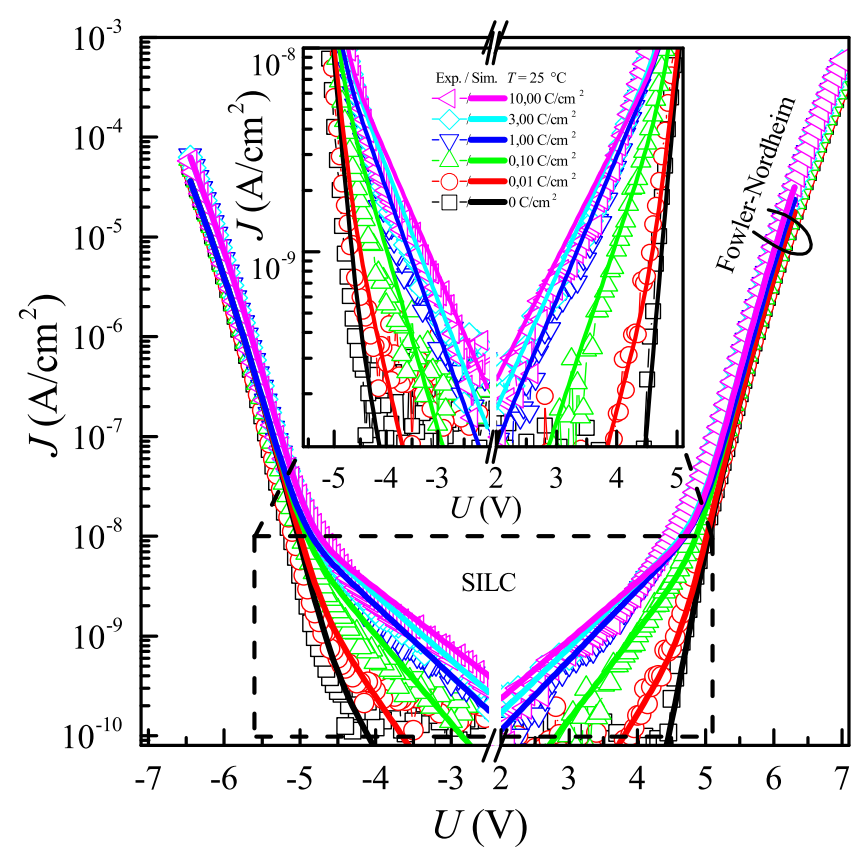

FIG. 2: (Color online) Experimental (characters) and calculated (lines) current-voltage characteristics of $n$-Si $/ \mathrm{SiO}_{2} /$ poly-Si structures at room temperature $\left(25^{\circ} \mathrm{C}\right)$ after different SILC stress. Calculations were done using Eq. (6). The inset: SILC mode.

rent through $\mathrm{SiO}_{2}$ films is limited by Fowler-Nordheim tunneling. The current at lower voltages $|U|<5 \mathrm{~V}$ is controlled by SILC. The characters in Fig. 2 represent the experimental $J-V$ characteristics of $n$ - $\mathrm{Si} / \mathrm{SiO}_{2} /$ poly$\mathrm{Si}$ structures at room temperature after different SILC stresses. The SILC currents $(2.5 \mathrm{~V}<|U|<5 \mathrm{~V})$ grow with the increasing SILC stress. The slope of $J-V$ curves at low voltages decreases if the SILC is increased. The temperature increase leads to growing SILC currents on 30-50\%, but Fowler-Nordheim tunneling.

When the trap density is high and the distance between them is short enough, trapped electrons can tunnel between the neighboring traps without ionization to the conduction band ${ }^{28}$.

A diagram of the electron tunneling from a phononbound trap to the other one at a distance of $a$ in the presence of an external electric field is shown in Fig. 3. The energy dependencies from generalized coordinate $Q$ of a system trapped-electron-bound-with-phonon are shown by $U_{\mathrm{b}}(Q)$ terms. The $U_{\mathrm{f}}(Q)$ terms correspond to "free" electrons in the conduction band. Solid lines represent the initial, before the tunneling state, dashed lines represent the final state after tunneling. Due to the external electric field electrons on the neighboring traps have different energy levels (slanted lines $\varepsilon(Q)$ ), and the tunnel transition must be accompanied by inelastic processes, like phonon emission and phonon absorption, in order to compensate the energy difference. The phonon-assisted tunneling model ${ }^{28}$ takes into account this circumstance. 


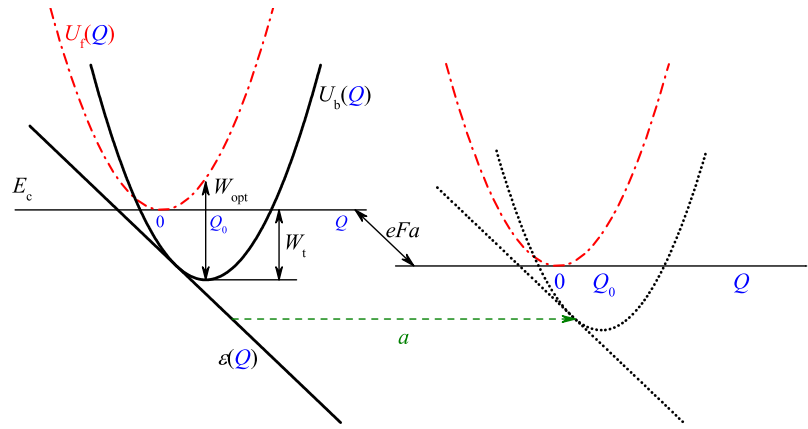

FIG. 3: (Color online) Configuration diagram for two phonon-coupled traps. $U_{\mathrm{f}}(Q)$ is the potential energy of an empty oscillator (without trapped electron); $U_{\mathrm{b}}(Q)$ is the potential energy of an occupied oscillator (with trapped electron); $\varepsilon(Q)$ is the position of the energy level of the trapped electron dependent on coordinate $Q ;$ and $E_{\mathrm{c}}$ is the conduction-band edge. The solid and dotted lines refer to the initially occupied and empty

state, respectively. The most probable tunneling transition for the electron when both oscillators take position $Q_{0} / 2$ is shown by the horizontal dashed arrow.

According to this model, the rate of such transitions is given by

$$
\begin{aligned}
P_{\text {tun }} & =\frac{2 \sqrt{\pi} \hbar W_{\mathrm{t}}}{m^{*} a^{2} Q_{0} \sqrt{k T}} \exp \left(-\frac{2 a \sqrt{2 m^{*} W_{\mathrm{t}}}}{\hbar}\right) \times \\
& \times \exp \left(-\frac{W_{\mathrm{opt}}-W_{\mathrm{t}}}{2 k T}\right) \sinh \left(\frac{e F a}{2 k T}\right) .
\end{aligned}
$$

Here $\hbar=h / 2 \pi, Q_{0}=\sqrt{2\left(W_{\mathrm{opt}}-W_{\mathrm{t}}\right)}, W_{\mathrm{t}}$ and $W_{\mathrm{opt}}$ are thermal and optical trap energies, $k$ in the Boltzmann constant, $T$ is the temperature. At high temperatures, the charge transport through the dielectrics is described within monopolar model involving Shockley-Read-Hall equations and the Poisson equation:

$$
\begin{gathered}
\frac{\partial n_{\mathrm{t}}}{\partial t}=\frac{1}{e} \boldsymbol{\nabla} \cdot \boldsymbol{J}-a \boldsymbol{\nabla} \cdot\left(n_{\mathrm{t}}\left(1-\frac{n_{\mathrm{t}}}{N}\right) P_{\mathrm{tun}} \frac{\boldsymbol{F}}{|\boldsymbol{F}|}\right), \\
\boldsymbol{\nabla} \cdot \boldsymbol{F}=-\frac{e n_{\mathrm{t}}}{\varepsilon \varepsilon_{0}},
\end{gathered}
$$

where $n_{\mathrm{t}}$ is the filled trap density, $t$ is time, $N=a^{-3}$ is the trap density, $J$ is the current density, $\varepsilon$ is the static $\mathrm{SiO}_{2}$ permittivity $(\varepsilon=3.9), \varepsilon_{0}$ is the electric constant. In the static one-dimensional case, Eq. (4) gives the currentvoltage characteristics

$$
J_{\text {tun }}=\frac{e}{a^{2}} \frac{n_{\mathrm{t}}}{N}\left(1-\frac{n_{\mathrm{t}}}{N}\right) P_{\mathrm{tun}} .
$$

Note, the tunneling current takes a maximum value at $n_{\mathrm{t}} / N=1 / 2$. Taking into account Fowler-Nordheim tunneling (2), one can get the total current-voltage characteristics

$$
J=\frac{s}{S} J_{\mathrm{tun}}+J_{\mathrm{FN}}
$$

where $J$ is the total current density, $S$ is the total sample square $\left(8 \times 10^{4} \mu \mathrm{m}^{2}\right), s$ is the square of SILC area. We assume that charge stress induces extra traps on a part of the $\mathrm{SiO}_{2}$ layer. This stressed part, having effective square of $s$, shunts the whole sample, and gives additive to the current.

The comparison of experimental data (characters) with the theory (6) (lines) is presented in Fig. 2. As a result, we obtained thermal and optical trap energies in $\mathrm{SiO}_{2}$ $W_{\mathrm{t}}=1.6 \mathrm{eV}$ and $W_{\mathrm{opt}}=3.2 \mathrm{eV}$, respectively. Simulations of experimental current-voltage characteristics measures at $70^{\circ} \mathrm{C}$ gives the same trap energy values. Simulations within well-known Frenkel model of isolated Coulomb center ionization ${ }^{29,30}$ predict that dinamic permittivity increases with the charge stress up to $\varepsilon_{\infty}=30$, that is physically incorrect results. All above confirm that using the model of the phonon-assisted tunneling between traps is correct. The obtained model parameter values including trap densities $N$ and the squares of SILC area $s$ depending on the total SILC charge $\Sigma$ are given in Table I.

We should note that the multi-phonon charge transport model in the SILC mode was introduced earlier ${ }^{16,17}$. This model is not analytical, and it requires complex numerical calculations to describe the SILC transport.

We assume that oxygen vacancies ( $\mathrm{Si}-\mathrm{Si}$ bonds) act as traps responsible for SILC in $\mathrm{SiO}_{2}$, which are generated as

$$
S i-O-S i->S i-S i+O
$$

where $\mathrm{O}$ is the interstitial oxygen atom. The electronic structure of $\mathrm{Si}-\mathrm{Si}$ bonds in $\mathrm{SiO}_{2}$ has been studied intensively ${ }^{4,8,9,11}$. The trap energies for electrons and holes, calculated from the first principles (1), are $1.2 \mathrm{eV}$ and $1.6 \mathrm{eV}$, respectively. The $\mathrm{Si}-\mathrm{Si}$ bond in silicon oxide acts as an amphoteric trap capable of both electron and hole capturing. Taking into account that such calculations usually underestimate electron trap energy values, one can conclude that the ab initio simulation results agree to the thermal trap energy value of $1.6 \mathrm{eV}$ obtained from transport simulations.

It is interesting to compare thermal trap energy $W_{\mathrm{t}}=$ $1.6 \mathrm{eV}$ in $\mathrm{SiO}_{2}$ to the Stokes shift of $\mathrm{Si}-\mathrm{Si}$ defect luminescence. It is known that $\mathrm{Si}-\mathrm{Si}$ bonds exhibit the ultraviolet luminescence band with the photon energy of $4.4 \mathrm{eV}^{9,31}$. The excitation maximum of this band is located at $7.6 \mathrm{eV}^{8,9,31}$. A half of the Stokes luminescence shift $(7.6-4.4) / 2=1.6 \mathrm{eV}$ is equal to the energy of electron and hole traps in $\mathrm{SiO}_{2}$, which are $\mathrm{Si}-\mathrm{Si}$ bonds ${ }^{11}$. This value is equal to the $W_{\mathrm{t}}$ value obtained from charge transport in $\mathrm{SiO}_{2}$ experiments in the SILC mode. Thus, one can conclude that, namely, oxygen vacancies act as the charge traps in $\mathrm{SiO}_{2}$ after the SILC stress.

Note that the obtained optical energy value $W_{\text {opt }}$ is twice bigger than thermal trap energy $W_{\mathrm{t}}$. This empirical rule of the multiphonon transport mechanism is executed for other dielectrics ${ }^{27,32-35}$. To confirm or refute universal nature of this correlation, it is necessary to collect 
TABLE I: Obtained values of parameter (2), (3), (5), (6).

\begin{tabular}{c|cccccc}
\hline \hline$\Sigma\left(\mathrm{C} / \mathrm{cm}^{2}\right)$ & 0 & 0.01 & 0.1 & 1 & 3 & 10 \\
\hline$W_{\mathrm{t}}(\mathrm{eV})$ & \multicolumn{5}{c}{1.6} \\
$W_{\text {opt }}(\mathrm{eV})$ & \multicolumn{5}{c}{3.2} \\
$m^{*} / m_{\mathrm{e}}$ & \multicolumn{6}{c}{$0.5 \pm 0.02$} \\
$N\left(\mathrm{~cm}^{-3}\right)$ & $\ll 10^{20}$ & $1 \times 10^{21}$ & $2 \times 10^{21}$ & $4 \times 10^{21}$ & $5 \times 10^{21}$ & $7 \times 10^{21}$ \\
$s\left(\mathrm{~nm}^{2}\right)$ & 0 & $25 \times 10^{2}$ & $1 \times 10^{4}$ & $1.5 \times 10^{4}$ & $2 \times 10^{4}$ & $3 \times 10^{4}$ \\
\hline \hline
\end{tabular}

statistics for a large number of dielectrics. The optical trap energy $W_{\text {opt }}=3.2 \mathrm{eV}$ evaluated from the transport measurement is very close to the electron trap energy of $3.1 \mathrm{eV}$ defined from the tunneling discharge of trapped in $\mathrm{SiO}_{2}$ electrons ${ }^{36}$.

Taking into account arguments above, one can conclude that namely oxygen vacancies are responsible for the SILC phenomenon, but not other defects in $\mathrm{SiO}_{2}$.

After the SILC stress some samples were treated by annealing at the temperature of $250^{\circ} \mathrm{C}$ during 120 hours. A comparison of the current-voltage characteristics measures for the "as deposited", after the SILC stress and after annealing structures are shown in Fig. 1. One can see that $J-V$ curves, after annealing, are almost identical to the "as deposited" control ones. This phenomenon demonstrates that long-time annealing leads to the electronic structure of the tunnel $\mathrm{SiO}_{2}$, which is identical to one of the "as deposited" films. This means that the trap density decreases with annealing down to initial values. This phenomenon makes it possible to controllably suppress the leakage currents caused by the tunnel $\mathrm{SiO}_{2}$ degradation due to the SILC stress.

In conclusion, the electron transport due to the traps in $\mathrm{SiO}_{2}$ was studied experimentally. It was demonstrated that the transport is limited by phonon-assisted tunneling between traps. Comparison trap energy values obtained from different experiments on charge transport in $\mathrm{SiO}_{2}$, luminescence of oxygen vacancy ( $\mathrm{Si}-\mathrm{Si}$ bond) in $\mathrm{SiO}_{2}$, and ab initio simulations of $\mathrm{Si}-\mathrm{Si}$ bond electronic structure, it was found that the oxygen vacancies $(\mathrm{Si}-\mathrm{Si}$ bonds) act as the charge localization centers (traps) in $\mathrm{SiO}_{2}$. The SILC stress leads to oxygen vacancies generation. The thermal and optical trap energies were evaluated. Long-time annealing at $250^{\circ} \mathrm{C}$ results in the recombination of oxygen vacancies and interstitial oxygen, and reduces the leakage current to the initial level.

This work was supported by the Russian Science Foundation (grant \#16-19-00002). The computations have been carried out at the computational cluster in the Rzhanov Institute of Semiconductor Physics SB RAS.

${ }^{1}$ M. Boero, A. Pasquarello, J. Sarnthein, and R. Car, Physical
Review Letters 78, 887 (1997).
${ }^{2}$ A.-M. El-Sayed, M. B. Watkins, T. Grasser, V. V. Afanas'ev,
and A. L. Shluger, Physical Review Letters 114, 115503 (2015).
${ }^{3}$ A.-M. El-Sayed, Y. Wimmer, W. Goes, T. Grasser, V. V.
Afanas'ev, and A. L. Shluger, Physical Review B 92, 014107
(2015).
${ }^{4}$ E. P. O'Reilly and J. Robertson, Physical Review B 27, 3780 (1983).
${ }^{5}$ C.-L. Kuo and G. S. Hwang, Physical Review Letters 97, 066101 (2006).

${ }^{6}$ L. Giordano, P. V. Sushko, G. Pacchioni, and A. L. Shluger, Physical Review B 75, 024109 (2007).

${ }^{7}$ S. Agnello, R. Boscaino, M. Cannas, F. M. Gelardi, M. Leone, and B. Boizot, Physical Review B 67, 033202 (2003).

${ }^{8}$ R. Tohmon, Y. Shimogaichi, H. Mizuno, Y. Ohki, K. Nagasawa, and Y. Hama, Physical Review Letters 62, 1388 (1989).

${ }^{9}$ G. Pacchioni and G. Ieranò, Physical Review Letters 79, 753 (1997).

${ }^{10}$ L. Giordano, P. V. Sushko, G. Pacchioni, and A. L. Shluger, Physical Review Letters 99, 136801 (2007).

${ }^{11}$ V. Gritsenko and H. Wong, Critical Reviews in Solid State and Materials Sciences 36, 129 (2011).

${ }^{12}$ P. V. Sushko, S. Mukhopadhyay, A. M. Stoneham, and A. L. Shluger, Microelectronic Engineering 80, 292 (2005).

${ }^{13}$ R. H. Fowler and L. Nordheim, Proceedings of the Royal Society of London A: Mathematical, Physical and Engineering Sciences 119, 173 (1928).

${ }^{14}$ S. M. Sze and K. K. Ng, Physics of Semiconductor Devices, 3rd ed. (Wiley, New York, 2006) p. 832.

${ }^{15}$ P. E. Blöchl and J. H. Stathis, Physical Review Letters 83, 372 (1999).

${ }^{16}$ T. Endoh, T. Chiba, H. Sakuraba, M. Lenski, and F. Masuoka, Journal of Applied Physics 86, 2095 (1999).

${ }^{17}$ F. Jiménez-Molinos, A. Palma, F. Gámiz, J. Banqueri, and J. A. López-Villanueva, Journal of Applied Physics 90, 3396 (2001).

${ }^{18} \mathrm{~K}$. Komiya and Y. Omura, Journal of Applied Physics 92, 2593 (2002).

${ }^{19}$ P. Giannozzi, S. Baroni, N. Bonini, M. Calandra, R. Car, C. Cavazzoni, D. Ceresoli, G. L. Chiarotti, M. Cococcioni, I. Dabo, A. Dal Corso, S. de Gironcoli, S. Fabris, G. Fratesi, R. Gebauer, U. Gerstmann, C. Gougoussis, A. Kokalj, M. Lazzeri, L. Martin-Samos, N. Marzari, F. Mauri, R. Mazzarello, S. Paolini, A. Pasquarello, L. Paulatto, C. Sbraccia, S. Scandolo, G. Sclauzero, A. P. Seitsonen, A. Smogunov, P. Umari, and R. M. Wentzcovitch, Journal of Physics: Condensed Matter 21, 395502 (2009).

${ }^{20}$ R. Williams, Physical Review 140, A569 (1965).

${ }^{21}$ See supplementary material http://dx.doi.org/10.1063/1.4960156 for the detailed description of the numeric simulation procedure.

${ }^{22}$ A. X. Chu and W. B. Fowler, Physical Review B 41, 5061 (1990).

${ }^{23}$ M. Petersen and Y. Roizin, Applied Physics Letters 89, 053511 (2006).

${ }^{24}$ A. S. Foster, F. Lopez Gejo, A. L. Shluger, and R. M. Nieminen, Physical Review B 65, 174117 (2002).

${ }^{25}$ V. A. Gritsenko, K. P. Mogil'nikov, and A. V. Rzhanov, JETP Letters 27, 375 (1978).

${ }^{26}$ Z. A. Weinberg, Journal of Applied Physics 53, 5052 (1982).

${ }^{27}$ K. A. Nasyrov, S. S. Shaimeev, V. A. Gritsenko, and J. H. Han, Journal of Applied Physics 105, 123709 (2009).

${ }^{28}$ K. A. Nasyrov and V. A. Gritsenko, Journal of Applied Physics 109, 093705 (2011).

${ }^{29}$ J. Frenkel, Technical Physics of the USSR 5, 685 (1938).

${ }^{30}$ J. Frenkel, Physical Review 54, 647 (1938).

${ }^{31}$ C. M. Gee and M. Kastner, Physical Review Letters 42, 1765 (1979).

${ }^{32}$ N. Novikov, V. A. Gritsenko, and K. A. Nasyrov, Applied 
Physics Letters 94, 222904 (2009).

${ }^{33}$ T. V. Perevalov, O. E. Tereshenko, V. A. Gritsenko, V. A. Pustovarov, A. P. Yelisseyev, C. Park, J. H. Han, and C. Lee, Journal of Applied Physics 108, 013501 (2010).

${ }^{34}$ T. V. Perevalov, V. S. Aliev, V. A. Gritsenko, A. A. Saraev, V. V. Kaichev, E. V. Ivanova, and M. V. Zamoryanskaya, Applied
Physics Letters 104, 071904 (2014).

${ }^{35}$ D. R. Islamov, V. A. Gritsenko, C. H. Cheng, and A. Chin, Applied Physics Letters 105, 222901 (2014), arXiv:1409.6887 [condmat.mtrl-sci].

${ }^{36} \mathrm{~K}$. Yamabe and Y. Miura, Journal of Applied Physics 51, 6258 (1980). 